L. Pearsonii übrig bliebe. Schon aus diesem Grunde dürfte diese Lösung der Frage ziemlich ausgeschlossen sein.

Noch eine dritte Möglichkeit möchte ich endlich andeuten, nämlich unsere Pflanze als eigene Art aufzufassen, die sich zwischen $L$. Pearsonii und L. reptans einschiebt, die dann den bezeichnenden Namen Lepidozia intermedia führen könnte. - Jedenfalls wird es aber geraten sein, erst abzuwarten, bis wir die interessante Pflanze von mehr Standorten kennen, dann wird es sich von selbst ergeben, für welche Deutung derselben wir uns entscheiden müssen.

\title{
Einige Planktonfänge aus dem Brenn-See bei Feld in Kärnten.
}

\author{
Von Dr. Karl von Keißler (Wien).
}

In den folgenden Zeilen möchte ich über die Ergebnisse einiger Planktonfänge berichten, die ich im Sommer 1903 dem Brenn-See bei Feld unweit Villach in Kärnten entnahm. Dieser kleine, schwarz gefärbte See liegt in einer Meereshöhe von $742 \mathrm{~m}$; sein Abfluß, der sog. "Feldbach", ergießt sich nach seiner Vereinigung mit dem "Tieferbach" als "Riegerbach" in den Millstätter See. Die Ufervegetation, nicht sehr reich entwickelt, besteht fast nur aus Phragmites communis L., die Ufer selbst gehen in sumpfige Wiesen aus. Das Wasser dieses Sees ist trotz der verhältnismäßig hohen Lage auffallenderweise warm; so beträgt die Oberflächentemperatur im Sommer im Durchschnitte ca. $20-24^{\circ} \mathrm{C}$.

Die Planktonfänge, von denen hier die Rede sein soll, wurden am 17. August 1903 ausgeführt (4 Uhr nachmittags, bewölkt, windig, leichte Wellen, Luft $14^{\circ} \mathrm{C}$., Wasseroberfläche $20^{\circ} \mathrm{C}$., Netz bis $4 \mathrm{~m}$ sichtbar). Hierbei ergab sich, daf das Plankton bis zu einer Tiefe von $10 \mathrm{~m}^{1}$ ) aus folgenden Organismen zusammengesetzt sei:

Chroococcus minutus Naeg. Selten.

Anabaena flos aquae Bréb. Selten. Sporenballen, hin und wieder einzelne Fadenstücke.

Anabaena macrospora Kleb. in Flora Bd. 80 (1895), p. 269, Tab. IV, Fig. 16-18. Sehr selten. Fäden einzeln, gerade, ca. $200 \mu$ lang, Zellen rundlich, halbiert, $6 \mu$ im Durchmesser, Heterocysten von gleicher Größe. Da keine Sporen wahrzunehmen sind, ist die Bestimmung nicht ganz sicher.

Coelosphaerium aerugineum (?) Lemm. in Botan. Zentralbl. Bd. 76 (1898), S. 154. Mäßig häufig. Nach der Beschreibung dürften die Exemplare mit $C$. aerugineum Lemm. identisch sein.

1) Es sei übrigens bei dieser Gelegenheit darauf verwiesen, daß in einer Anzahl von Seen Fänge nach größeren Tiefen keinerlei Organismen ergaben, die nicht in den Fängen von $10 \mathrm{~m}$ Tiefe enthalten wären. 
Coelosphaerium minutissimum (?) Lemm. in Ber. d. Deutsch. bot. Ges. Bd. 18 (1900), p. 309. Selten. Durchmesser der Kolonien $30 \mu$, Zellen $1 \mu$ im Durchmesser; manchmal sind um die Zellen schmale Gallerthüllen, zuweilen sind auch zwei Zellen in einer solchen Gallerthülle vereinigt.

Ceratium hirundinella O. F. M. Häufig. Ziemlich schlank e Exemplare, im Mittel $165=54 \mu$ lang, dr eihörnig, selten ein viertes Horn angedeutet; das seitliche Horn meist ziemlich lang, $45 \mu$ im Durchschnitt messend, oft nicht viel kürzer als das apikale Horn der hinteren Hälfte.

Peridinium cinctum Ehrbg. Selten. Manchmal runde cystenartige Gebilde, $20 \mu$ im Durchmesser, zu sehen.

Asterionella formosa Hassk. var. gracillima Grun. Sehr häufig und dominierend. Meist achtstrahlige Sterne, Durchmesser derselben ca. $150 \mu$.

Tabellaria flocculosa Kuetz. Sehr selten (wohl nur zufällig in das Plankton verschlagen).

Cosmarium bioculatum Bréb. Sehr selten.

Oocystis solitaria Wittr. Selten. Zellen $18 \mu$ lang, einzeln.

Sphaerocystis Schröteri Chod. Sehr selten. In Formen, wie sie Chodat im Bull. de l'herb. Boiss. Tom. V (1897), Pl. 9, Fig. 2, 4, 8, 11, 12 abbildet.

Als Verunreinigung sind zu nennen: Koniferenpollen, Pollenkörner einer Campanula-Art (mit stacheliger Exine), ferner nicht näher bestimmbare Pollenkörner (mit wabiger Struktur bei glatter Exine), sämtlich vereinzelt auftretend ${ }^{\mathbf{1}}$ ).

Aus obiger Tabelle ist zu ersehen, daß das Phytoplankton des Brenn-Sees zur angegebenen Zeit aus zwölf Arten sich zusammensetzt, von denen aber nur zwei, nämlich eine Asterionella-Art und Ceratium hirundinella O. F. M. in Rücksicht auf Häufigkeit des Vorkommens von Bedeutung sind. Einigermaßen von Wichtigkeit sind sonst nur noch die Chroococcaceen. Speziell Asterionella tritt in ungeheuerer Menge auf, was deshalb auffällig ist, weil in diversen anderen Seen der Alpen die Beobachtung gemacht wurde, daß Asterionella im Mai und Juni ein Maximum erreicht und von da an Menge rasch abnimmt. Ich selber habe im Plankton mehrerer Alpenseen in den Monaten Juli und August Asterionella immer nur in recht spärlicher Zahl

1) Das Zooplankton enthält folgende Organismen: Cyclops spec., Diaptomus spec., beide selten; Daphnia spec., Bosmina spec. beide sehr selten (vereinzelt auch Eier und Larvenzustände bemerkbar). Polyarthra platyptera Huds., Anuraea cochlearis Gosse, beide selten; Chromogaster spec., Mastigocerca capucina Wierc. et Zach. sehr selten. Vorticella spec. auf Anabaena aufsitzend, mit jener Art übereinstimmend, die ich im Wolfgang-See, ebenfalls auf Anabaena aufsitzend, beobachtet habe. (Vgl. Verh. d. k, k. zool.-botan. Ges. Wien. Bd. LII (1902), p. 312 u. 313); ferner eine Vorticellide, auf Krustaceen teils mit, teils ohne Stiel aufsitzend, manchmal auch losgelöst und freischwebend. Im allgemeinen spielt das Zooplanktongegenüber dem Phytoplankton eine sehr untergeordnete Rolle. 
vertreten gefunden. Hervorgehoben zu werden verdient ferner die Tatsache, daß im Plankton des Brenn - Sees zur genannten Zeit Dinobryon, Fragilaria und Cyclotella völlig fehlen.

In Rücksicht auf die Art der Verteilung der wichtigeren Phytoplanktonten in den Schichten ergab sich:

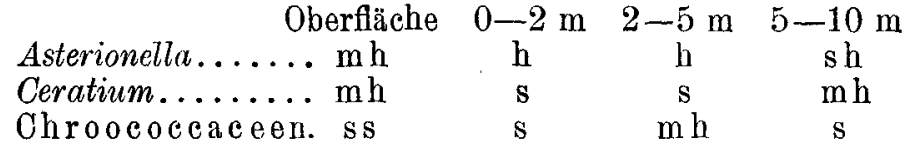

(Es bedeutet $\mathrm{h}=$ häufig, $\mathrm{mh}=$ mäßig häufig, $\mathrm{s}=$ selten, $\mathrm{ss}=$ sehr selten.)

Ferner wurden mit dem kleinen Apstein schen quantitativen Planktonnetz drei Stufenfänge nach einer Tiefe von $10 \mathrm{~m}$ ausgeführt, die als Mittelwert folgende Zahlen lieferten:

$$
\begin{array}{ccccc}
0-2 \mathrm{~m} & \text { Tiefe } & 0.06 \mathrm{~cm}^{3} & \text { Plankton gefischt } \\
0-5 " & 0.17 & " & \\
0-10 " & 0.28 & &
\end{array}
$$

Die Planktonmenge ist im Vergleiche zu einem anderen kleinen See, den ich untersuchte, nämlich dem Nussen - See ${ }^{3}$ ) in Oberösterreich (609 m Seehöhe, mit niederer Wassertemperatur), eine geringe. Proben aus demselben ergaben nämlich (31. August 1902) für die Tiefe von $10 \mathrm{~m}$ eine Planktonmenge von $0.42 \mathrm{~cm}^{3}$. Das trotz der auffallenden Wärme des Wassers des Brenn-Sees geringe Planktonquantum ist wohl auf das im Gegensatze zum Nussen-See spärliche Vorhandensein von Krustaceen zurückzuführen; denn diese sind es ja meistens, welche bei der im Vergleich zu den Phytoplanktonten bedeutenden Größe der Individuen für das Planktonquantum von ausschlaggebender Bedeutung sind.

Für die einzelnen Schichten ergeben sich aus den oben stehenden Zahlen durch Subtraktion bei gleichzeitiger Redultion der Planktonmenge auf $1 \mathrm{~m}$ innerhalb der betreffenden Schichte ${ }^{4}$ ) folgende Werte:

$0-2 \mathrm{~m} 0.03 \mathrm{~cm}^{3}$ Plankton $=4560 \mathrm{~mm}^{3}$ Plankton unter $1 \mathrm{~m}^{2}$

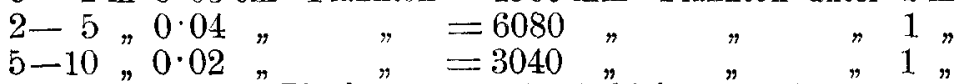

Setzt man die Planktonmenge der Schichte von $5-10 \mathrm{~m}=1$, so bekommt man folgende Verhältniszahlen:

$$
\begin{aligned}
& 0-2 \mathrm{~m}=1 \cdot 5 \\
& 2-5 \%=2 \\
& 5-10 \%=1
\end{aligned}
$$

Daraus folgt, daß zur angegebenen Tages- und Jahreszeit das Maximum in der Schichte von 2-5 $\mathrm{m}$ gelegen zu sein scheint.

3) Ygl. Österreichische botanische Zeitschrift, Bd. LII (1902), p. $6 \mathrm{ff}$.

4) Über diese Umrechnungen siehe Apstein, Das Sübwasserplankton Kiel, 1896), S. 68; vgl. auch K i ßler, l. c. S. 7. 